\title{
The onset of rheumatoid arthritis following
}

\section{trauma}

\section{Arthur E Brawer \\ Noopur Goel}

Department of Medicine, Monmouth Medical Center, Long Branch, NJ, USA
Correspondence: Arthur E Brawer Division of Rheumatology, Department of Medicine, Monmouth Medical Center, 300 Second Avenue, Long Branch, NJ 07740, USA

Tel +17328703133

Fax + I 7322220824

Email arthurbrawer@optimum.net
This article was published in the following Dove Press journal:

Open Access Rheumatology: Research and Reviews

17 August 2016

Number of times this article has been viewed

Background: Rheumatoid arthritis (RA) is known to have many predisposing factors.

Objective: We studied individuals whose RA was initiated by physical injuries.

Patients and methods: Sixty patients (43 females), previously well, developed RA following trauma. No other known environmental or familial influences were present. Fourteen sustained a fracture; of the 46 who did not, 36 sustained multiple injuries that in part involved the axial skeleton. Subsequent unremitting daily pain, stiffness, limited motion, pain on motion, and/or swelling in the injured areas were mandatory for inclusion.

Results: Nine months after injuries (span: 2 weeks-36 months), more obvious signs of inflammation (IM) appeared in multiple other joints that were previously not affected by the original trauma. In those with laboratory tests done prior to the spread of IM (30/60), 22 (73\%) were normal until an average 8 months after the spread of IM. Of the entire cohort of 60 , only $23 \%$ had a positive rheumatoid factor, but $43 \%$ had a positive antinuclear antibody.

Conclusion: It seems apparent that any severe trauma to a joint may precipitate an ongoing localized chronic inflammatory disorder for an indefinite period of time, which may then lead to the spread of IM to multiple other joints. The initiation of RA following trauma warrants consideration as a legitimate entity.

Keywords: rheumatoid arthritis, trauma, injuries, inflammation, antinuclear antibody, rheumatoid factor

\section{Introduction}

The relationship between physical trauma and the subsequent appearance of rheumatoid arthritis (RA) remains a subject of considerable controversy despite numerous publications and case reports on this topic. ${ }^{1-5}$ It is generally accepted that physical injuries can readily aggravate a preexisting arthritic condition, but the initiation of chronic systemic inflammation (IM) following trauma in a previously normal person is more difficult to substantiate.

Although the clinical manifestations and pathology of RA are well known, its cause remains unknown. Observations that RA can begin following a variety of different infections, severe emotional upset, or various vaccinations coexist with environmental risk factors such as insecticides, pesticides, smoking, periodontitis, hypoxia, hormonal imbalances, and prolonged exposure to unusual temperature changes. ${ }^{6-13}$ This suggests that other predisposing factors such as trauma may also be related to the onset of RA. We report on 60 cases where RA was initiated by a wide variety of physical injuries, 
including (singly or in combination): 1) obvious peripheral joint injuries with observable chronic IM; 2) fractures, with or without other structural abnormalities (eg, cartilage, ligament, and/or tendon disruptions; joint dislocation); and 3) structural or nonstructural trauma to the axial skeleton (spine, hips, pelvis, and shoulders).

\section{Patients and methods}

Sixty patients (43 females), aged $23-75$ years, with a variety of severe physical injuries comprised the study group. Of the 14 with fractures, there existed considerable diversity and multiplicity of sites, including both intra-articular and nonarticular areas. Thirty-six patients sustained trauma to diffuse areas of their spine, hips, pelvis, and shoulders, 90\% of which coexisted with other axial and/or peripheral bone, joint, ligament, cartilage, and tendon injuries. Ten patients sustained nonstructural peripheral joint trauma where obvious observable abnormalities on physical exam persisted unabated. Inclusion criteria for all patients mandated continuous uninterrupted daily pain, stiffness, limited motion, pain on motion, and/or swelling in the injured areas without resolution. None had prior phenomena of any inflammatory systemic connective tissue disease, spondyloarthropathy, or ongoing arthritis in the injured areas. None had a family history of RA, previous severe trauma, or any other known predisposing environmental factors as outlined in the "Introduction" section. Laboratory analyses predated the era of antibody testing to cyclic citrullinated peptides. The diagnosis of RA was based upon fulfillment of the American College of Rheumatology criteria in effect for the relevant years of patient presentation. Forty patients were self-referred and/ or physician referred, and 20 were referred by attorneys. Follow-up averaged 5 years.

Patients provided written informed consent for the synovial biopsy procedures. Monmouth Medical Center's ethics committee did not require that the authors obtain approval or further patient consents for this study.

\section{Results}

On the background of daily unremitting complaints and objective signs in the injured areas, more obvious signs of IM eventually appeared in multiple other small and large joints in 55/60 patients an average of 9 months from the original trauma (span: 2 weeks-36 months). Half of the entire cohort (30/60) were able to have blood tests drawn prior to the spread of IM to noninjured joints. These tests included routine blood counts and chemistries as well as rheumatoid factor (RF) via the sheep cell agglutination test, Westergren sedimentation rate (ESR), and antinuclear antibody (ANA) via indirect immunofluorescence. In 22/30 (73\%) patients, these latter three tests were normal or negative until an average 8 months had elapsed subsequent to the spread of IM to noninjured joints. Thus, on average, in this subgroup nearly 1 and $1 / 2$ years elapsed from the time of initial injuries until laboratory tests corroborated the patients' complaints.

One-hundred percent of the fracture group and $80 \%$ of the nonfracture group have at least one laboratory abnormality encompassing these latter three tests: $23 \%$ a positive RF; $43 \%$ a positive ANA (titer span: 1:80-1:320); and 52\% an elevated ESR (entire cohort span: 2-100). The nine patients with persistently normal laboratory readings were among the $31 / 60$ patients who had erosions in at least one joint and 8/60 who had synovial biopsies revealing chronic synovitis compatible with RA. One-third of all erosions were seen in joints initially injured, and two-thirds were present in noninjured joints. Synovial biopsies were equally divided between injured and noninjured joints. None of the abnormal synovial biopsies revealed germinal centers or granulomas, and no immunohistochemical studies were performed on these biopsies.

The definitive diagnosis of RA in the 60 patients was arrived at, on average, 24 months from the occurrence of the original injuries. All patients eventually manifested protracted morning stiffness lasting several hours, and 30/60 underwent detailed analysis of synovial fluid (average white blood cells =3,700). Extra-articular manifestations were infrequent. Only two patients developed rheumatoid nodules, and only two patients manifested Sjogren's syndrome. None developed vasculitis or rheumatoid lung disease. Despite permanence of their RA, severity within the entire cohort was quite variable and could not be correlated with the magnitude of injured areas. In one patient, an intra-articular fracture of a single joint led to severe RA, but in other instances multiple injuries were followed by a more indolent course. Five patients had persistent chronic IM only in the injured areas during their 5-year follow-up (three with radiographic erosions and two with chronic synovitis on biopsy). They are part of a subgroup of $8 / 30$ patients demonstrating abnormal laboratory results (ESR or sheep cell agglutination test or ANA) prior to IM spreading to noninjured joints. Thus, the observation of rapid serologic conversion did not necessarily herald the development of severe additional joint involvement. No differences exist between the 20 attorney-referred patients and the 40 other patients with regard to types of injuries, demographics, chronological evolution, presence or absence of laboratory abnormalities, follow-up time, or any other parameters. 


\section{Discussion}

Prior reports of physical injuries preceding the onset of RA have generated considerable controversy. Our results suggest that trauma can indeed initiate RA. We have shown that physical injuries are capable of producing chronic synovitis in previously healthy joints, with such a process then persisting for an indefinite period of time. This persistence of an uninterrupted traumatically initiated joint IM then carries a risk of subsequently triggering a more generalized systemic inflammatory process clinically indistinguishable from RA beginning spontaneously.

Multiple mechanisms that could produce such a condition have been studied. Furman et a ${ }^{14}$ reported acute IM after an articular fracture, which was evidenced by increased synovitis, increased macrophage infiltration into the synovium, and increased concentration of biomarkers in the synovial fluid and serum. Chronicity of some of these phenomena, extending over 5 years, has been reported by Struglics. ${ }^{15}$ Our observations of the chronological sequence of RA development after trauma are well within this timeline. Lindblad et $a l^{16}$ demonstrated similar immune histochemical patterns of synovial cell infiltrates in both RA and chronic traumatic synovitis. Other mechanisms capable of activating a variety of inflammatory pathways after trauma have been reported. These include localized or systemic hypoxia from tissue damage; $;{ }^{12}$ ischemia reperfusion $;{ }^{12}$ exposure of mitochondrial constituents and nuclear antigens from dying tissue ${ }^{17}$ and the impaction of cartilaginous particles into synovial tissue, which then induces the production of proteinases and inflammatory cytokines by synovial lining cells. ${ }^{18,19}$ This last mechanism may also have relevance for eliciting antibodies to Type II collagen, a phenomenon that some have implicated in the pathogenesis of RA. ${ }^{20}$ In aggregate, these mechanisms seem to suggest a different clinical phenotype for RA initiated by trauma, and they may provide an explanation for the low percentage of our patients developing a positive RF and a high percentage developing a positive ANA.

The incidence of RA following trauma (RAFT) remains to be elucidated. At a minimum, such a study would require a large multicenter cooperative effort by orthopedic surgeons and rheumatologists, adequate assessment of inflammatory markers, lengthy clinical follow-up, differentiation of mechanisms causing posttraumatic osteoarthritis from those triggering RAFT, and exclusion of other confounding predisposing factors. Even in the absence of such a study, our observations strengthen the conclusions of previous publications that the initiation of RAFT warrants consideration as a legitimate entity.

\section{Disclosure}

There have been no grants, no financial support, nor any other benefits from any commercial source for the work reported in this manuscript. The authors report no other conflicts of interest in this work.

\section{References}

1. Al-Allaf AW, Sanders PA, Ogston SA, Marks JS. A case control study examining the role of physical trauma in the onset of rheumatoid arthritis. Rheumatology. 2001;40:262-266.

2. Julkunen H, Rasanen JA, Kataja J. Severe trauma as an etiologic factor in rheumatoid arthritis. Scand J Rheumatol. 1974;3:97-102.

3. Boscarino JA, Forsberg CW, Goldberg J. A twin study of the association between PTSD symptoms and rheumatoid arthritis. Psychosom Med. 2010;72:481-486.

4. Barraud F, Gerster JC, Saudan Y. Post-traumatic rheumatoid polyarthritis and algodystrophy. Rev Med Suisse Romande. 1979;99:57-61.

5. Bouvenot G, Delboy C, Roux H, Serratrice G, Recordier AM. [Rheumatoid arthritis and trauma. 5 cases]. Rev Rhum Mal Osteoartic. 1976;43:619-624. French.

6. Masdottir B, Jonsson T, Manfreosdottir V, Vikingsson A, Brekkan A, Valdimarsson H. Smoking, rheumatoid factor isotypes and severity of rheumatoid arthritis. Rheumatology. 2000;39:1202-1205.

7. Kallberg H, Ding B, Padyukov L, et al. Smoking is a major preventable risk factor for rheumatoid arthritis: estimations of risks after various exposures to cigarette smoke. Ann Rheum Dis. 2011;70:508-511.

8. De Pablo P, Dietrich T, McAlindon TE. Association of periodontal disease and tooth loss with rheumatoid arthritis in the US population. J Rheumatol. 2008;35:70-76.

9. Katz, Warren A. Diagnosis and Management of Rheumatic Diseases. 2nd edition. Philadelphia, PA: JB Lippincott Company; 1988.

10. Wallace DJ. The role of stress and trauma in rheumatoid arthritis and systemic lupus lupus erythematosus. Semin Arth Rheum. 1987;16:153-157.

11. Symmons DP, Chakravarty K. Can immunization trigger rheumatoid arthritis? Ann Rheum Dis. 1993;52:843-844.

12. Eltzschig HK, Carmeliet P. Hypoxia and inflammation. $N$ Engl J Med. 2011;364:656-665.

13. Parks CG, Walitt BT, Pettinger $M$, et al. Insecticide use and risk of rheumatoid arthritis and systemic lupus erythematosus in the women's health initiative observational study. Arth Care Res. 2011;63:184-194.

14. Furman BD, Kimmerling KA, Zura RD, et al. Articular ankle fracture results in increased synovitis, synovial macrophage infiltration, and synovial fluid concentrations of inflammatory cytokines and chemokines. Arth Rheum. 2015;67:1234-1239.

15. Struglics A, Larrson S, Kumahashi N, Frobell R, Lohmander LS. Changes in cytokines and aggrecan ARGS neoepitope in synovial fluid and serum and in C-terminal crosslinking telopeptide of type II collagen and $\mathrm{N}$-terminal crosslinking telopeptide of type I collagen in urine over five years after anterior cruciate ligament rupture. Arth Rheum. 2015;67:1816-1825.

16. Lindblad S, Klareskog L, Hedfors E, Forsum U, Sundstrom C. Phenotypic characterization of synovial tissue cells in situ in different types of synovitis. Arth Rheum. 1983;26:1321-1332.

17. Manfredi AA, Rovere-Querini P. The mitochondrion - a Trojan horse that kicks off inflammation? N Engl J Med. 2010;362:2132-2134.

18. Sward P, Frobell R, Englund M, Roos H, Struglics A. Cartilage and bone markers and inflammatory cytokines are increased in synovial fluid in the acute phase of knee injury (hemarthrosis) - a cross-sectional analysis. Osteoarth Cartilage. 2012;20:1302-1308.

19. Evans C, Mears DC, Cosgrove JL. Release of neutral proteinases from mononuclear phagocytes and synovial cells in response to cartilaginous wear particles in vitro. Biochim Biophys Acta. 1981;677:287-294.

20. Trentham DE, Townes AS, Kang AH. Autoimmunity to type II collagen: an experimental model of arthritis. J Exp Med. 1977;146:857-868. 


\section{Publish your work in this journal}

Open Access Rheumatology Research and Reviews is an international, peerreviewed, open access journal publishing original research, reports, editorials, reviews and commentaries on all aspects of clinical and experimental rheumatology in the clinic and laboratory including the following topics: Pathology, pathophysiology of rheumatological diseases; Investigation, treatment and management of rheumatological diseases; Clinical trials and novel pharmacological approaches for the treatment of rheumatological disorders. The manuscript management system is completely online and includes a very quick and fair peer-review system, which is all easy to use. Visit http://www.dovepress.com testimonials.php to read real quotes from published authors. 